\title{
Audit and Feedback in emergency: A systematic review and an Italian project to investigate and improve quality of care
}

\author{
Sabrina Licata, Annarita Tullio, Francesca Valent \\ Istituto di Igiene ed Epidemiologia Clinica, Azienda Sanitaria Universitaria Integrata di Udine, Udine, Italy
}

\begin{abstract}
The Audit and Feedback process (A\&F) is commonly accepted as a good way to improve quality in health care, also in Emergency Departments (ED), where health aspects and pathologies are very different, usually acute and highly complex. Within an Italian Ministry of Health research project called EASY-NET, we conducted a systematic review of literature on A\&F in EDs from 2014 to December 2019 to evaluate the impact of this approach in a particular setting where time-dependent indicators are fundamental. We selected 24 articles: 9 about infective pathologies (i.e. antibiotic stewardship), 6 about cardiovascular acute emergencies (i.e. cardiac arrest), 2 about stroke, 3 about laboratory tests, and 4 about other fields (i.e. diabetic ketoacidosis or use of prothrombin complex). Most of articles proposed a multimodal approach: only 7 concerned A\&F alone. Despite the wide range on interventions modality and the poor comparability of the considered studies, the results are encouraging and confirm the importance to implement A\&F both in emergency and in other clinical settings.
\end{abstract}

Correspondence: Sabrina Licata, Istituto di Igiene ed Epidemiologia Clinica, Azienda Sanitaria Universitaria Friuli Centrale, Via Colugna 50, 33100, Udine, Italy.

Tel.: +39 3397392782

E-mail: sabrina.licata@uniud.it

Key Words: Audit and Feedback; Emergency Departments; TimeDependent Indicators; Interrupted Time Series.

Funding: This work was funded by the Italian Ministry of Health (Ministero della Salute) and co-funded by the participating Regions Lazio, Friuli Venezia Giulia, Piemonte, Emilia-Romagna, Lombardia, Calabria through the Programma di rete Ricerca Finalizzata, Bando 2016 - NET-2016-02364191.

Conflict of interest: The author declares no potential conflict of interest. Ethics approval and consent to participate: not applicable.

Received for publication: 21 June 2020.

Accepted for publication: 15 September 2020.

This work is licensed under a Creative Commons Attribution 4.0 License (by-nc 4.0).

(C) Copyright: the Author(s), 2020

Licensee PAGEPress, Italy

Emergency Care Journal 2020; 16:9201

doi:10.4081/ecj.2020.9201

\section{Background}

The use of Audit and Feedback (A\&F) is commonly accepted as a tool to improve quality in all fields of medicine. ${ }^{1}$ It can also be used to evaluate changes in care provision or to confirm that current practice meets the expected level of performance. ${ }^{2}$

A review made by Ivers et al. in 2012, about all medical fields, showed how the efficacy of intervention was higher when the baseline performance was low, the feedback was made by a supervisor or a colleague and was delivered in both oral and written formats with explicit indications on action plans and targets. ${ }^{3}$

In emergency care, audit can concern both medical practice (following guidelines, drug prescriptions etc.), or patient point of view (satisfaction, waiting time, etc.).

Even if many studies were published about that in the last 20 years, there is still lack of information and a not unique agreement about the importance and validity of A\&F in Emergency Departments (EDs). This maybe be due to the peculiarity of EDs environment, which involves many health aspects and pathologies, most of the time in acute high complexity presentation.

A review conducted by Rogers et al. (2014) based on A\&F in EDs concluded with positive results for interventions on different clinical conditions, but with not enough data for a standardized meta-analysis. ${ }^{4}$ This review included studies published till January 2014. With regard to A\&F, in 2019 Italian Ministry of Health approved a research project called Easy-Net (NET-201602364191), a network composed by 7 teams of seven different research centers each one dislocated in a different Italian Region (Lazio, Friuli Venezia Giulia, Piemonte, Emilia Romagna, Lombardia, Calabria). The aim of the project is to spread the knowledge of A\&F in different fields of Italian health system and to promote A\&F application thus increasing quality and access of health assistance. Each research center has a research sub-project based on specific clinical areas: cancer, emergency, gynecology, rehabilitation, etc. Our center, dislocated in Friuli Venezia Giulia, has a sub-project called Audit and feedback: efficacy for enhancing the clinical practice and to reduce avoidable differences in the field of emergency medicine, with particular regard to heart attack, stroke and trauma. Major details about the EASY-NET project can be found at https://easy-net.info/

Based on our sub-project, we performed a review of the literature on A\&F in EDs from 2014 to December 2019. Our main outcome was to identify how A\&F process can influence medical and, in general, health care people behavior in emergency.

\section{Methods}

This work was performed in adherence with the Preferred Reporting Items for Systematic Reviews and Meta-analyses statement. ${ }^{5}$ We performed a systematic review of literature starting from January 2014 to December 2019 on the major medical data- 
base: PubMed, Cochrane library, CINAHL, OVID clinical Edge. The research included all articles about A\&F in EDs. The searched terms are listed in Table 1.

Each screening passage has been made by 2 contemporarily reviewers, as described in Figure 1. When there was disagreement, the third author's opinion was taken into account for final decision.

We found 553 articles, identified through search on databases: 321 were duplicates or not pertinent, so we screened 232 abstracts, of whom 64 were chosen and relative full text were searched.

Six full text consisted in reviews and we decided to exclude them from our analysis and search for full texts of cited studies that were published between 2014 and 2019, if pertinent.

Screening of full texts led us to 24 articles that became the object of our analysis (indicated as "selected studies").

Studies were only included if they met the following eligibility/ inclusion criteria: i) the study used both A\&F, ii) the study was conducted in EDs, both exclusively or as a multidisciplinary intervention, iii) the study has clear results, both qualitative or quantitative, iv) the study was prospective.

All articles were published in English language. We also reviewed the reference lists of selected articles to identify additional studies for inclusion.

Due to the heterogeneity and sometimes to the lack of standardized methods used to perform statistical analysis in selected studies, we conducted a descriptive analysis of the reported results and a qualitative comparison of the different reported $A \& F$ approaches.

\section{Results}

We chose 24 articles, ${ }^{6-29}$ most of whom have been conducted in USA. All 24 studies followed the before/after scheme and 5 have a Plan-Do-Study-Act (PDSA) design. ${ }^{8-10,22}$ While 9 studies involved different medical departments, ${ }^{6,10-12,17,19,21,22,27} 15$ of them concerned only Emergency departments and/or paramedics. Most of articles proposed a multimodal approach: only 7 of them concerned exclusively A\&F. ${ }^{7-11,13,22}$ As it's shown in Table 2, 9 articles talked about measurements for infective pathologies (especially antibiotic stewardship), $, 8,12,13,16,17,21,22,296$ of them treated cardiac arrest or cardiovascular emergency problems, ${ }^{8,19,20,25-27} 2$ of them treated stroke, ${ }^{15,24}$ 3 ones were about laboratory analyses ${ }^{10,18,28}$ and 4 of them treated any other fields (diabetic ketoacidosis, use of a prothrombin complex, etc.), (Table 2). Based on A\&F approach, most of articles report a multimodal A\&F method. In 15 studies, there were multiple reports (basically weekly or monthly reports); meetings and frontal lessons were used in 18 studies; surveys were used in 6 studies; 9 studies used printed materials (leaflets, posters, hold in pocket charts); in 9 studies, there were other electronic methods
(Table 3). All studies declared an improvement due to the interventions.

\section{Discussion}

A\&F is a common accepted method to assess quality in healthcare organizations, ${ }^{1-4}$ alone or in combination with other interventions, to improve health professionals' performance and encourage the health care people in following professional standards.

The auditing consists in a measurement of an individual's (or specific group's) professional practice or performance and then in a comparison of the results to professional standards or targets. The results of this comparison are then sent to the individual or the group as a feedback. . $^{3,30,31}$

Even if there have been different attempts to standardize A\&F management and processing, ${ }^{32-34}$ there is still a lot of heterogeneity in literature concerning the correct methodology to collect and analyze data and for the choice of right indicators. The Italian Friuli Venezia Giulia region (FVG) takes part to a national project to evaluate the A\&F approach applied to different clinical areas. The FVG sub-project is related to emergency departments with a particular regard to big emergency situations as hearth attack (MI), stroke and major traumas. We conducted a review on main medical electronic databases to understand better the state of art on this topic. Our main outcome was to identify how A\&F process can influence medical and, in general, health care people behavior in the field of emergency. In general, we observed a great variability between the articles concerning pathologies or clinical aspects to

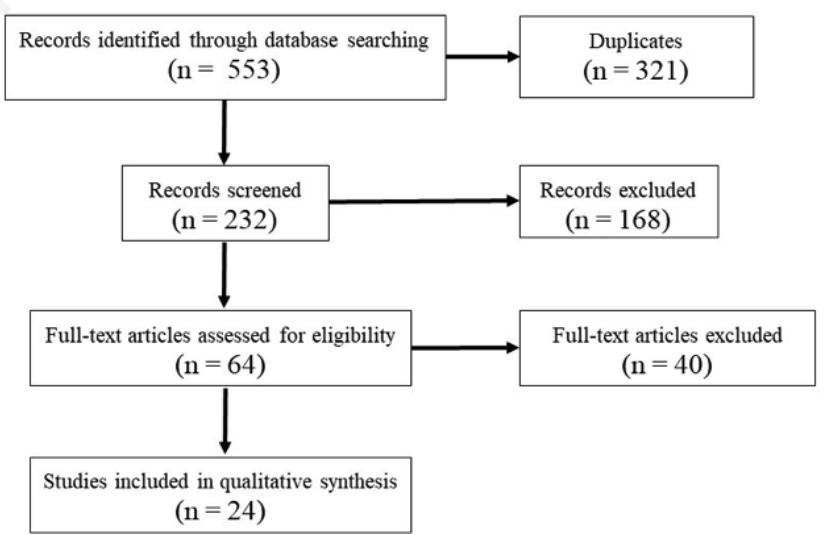

Figure 1. Flow chart of different research phases.

Table 1. terms used to perform research.

\begin{tabular}{lll}
\hline Audit & Feedlback & Audit and feedback \\
Audit emergency & feedback emergency & Audit and feedback emergency \\
Audit emergency department & Feedback emergency department & Audit and feedback emergency department \\
\hline Audit "emergency department" & Feedback "emergency department" & Audit and feedback "emergency department" \\
Audit emergency care & Feedback emergency care & Audit and feedback emergency care \\
\hline Audit "emergency care" & Feedback "emergency care" & Audit and feedback "emergency care" \\
Audit emergency unit & Feedback emergency unit & Audit and feedback emergency unit \\
\hline Audit "emergency unit" & Feedback "emergency unit" & Audit and feedback "emergency unit"
\end{tabular}


Table 2. selected studies listed by health field, year and country.

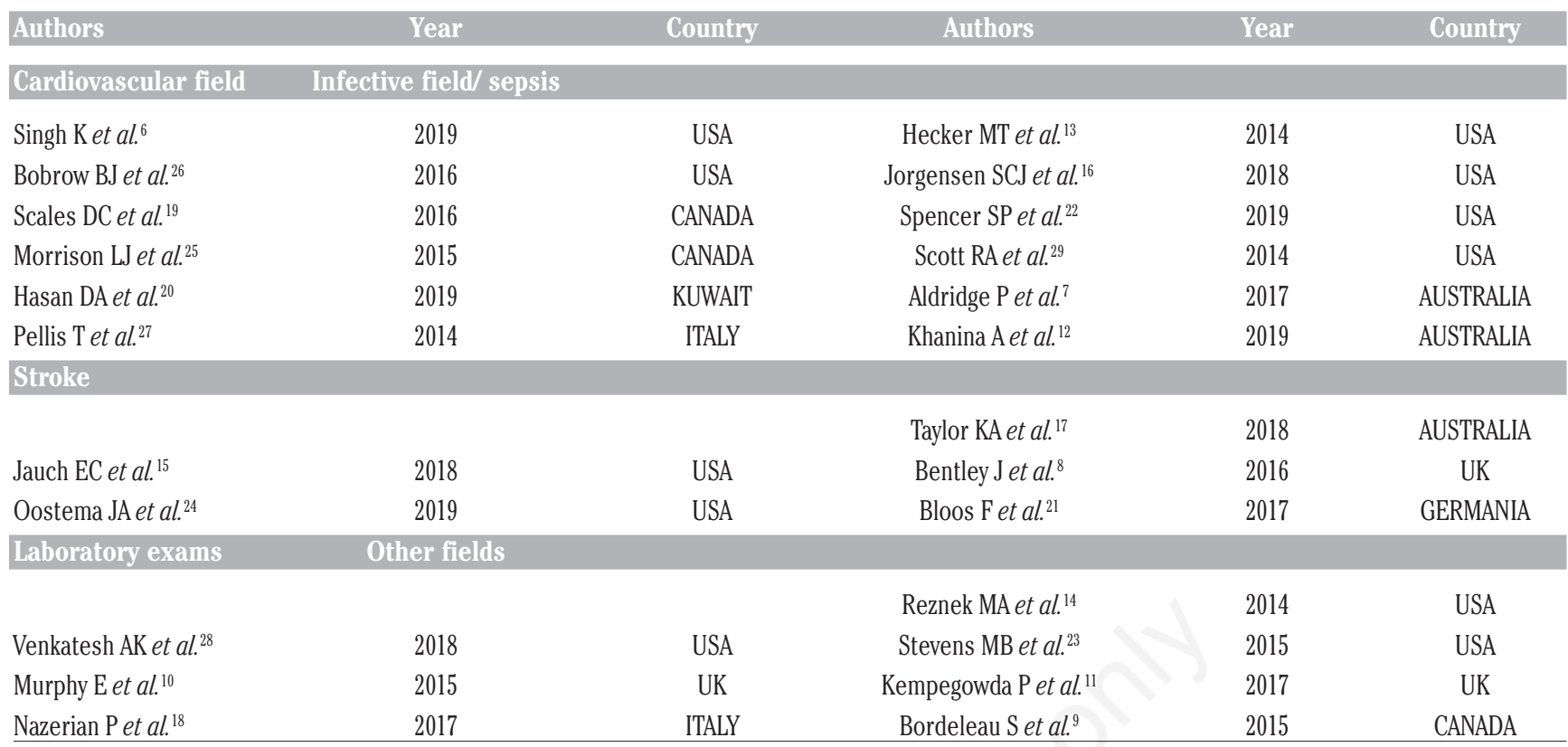

whom they were direct, involved departments, clinical figures and practitioners, countries in which studies were conducted and levels of assistance, type and methods of A\&F with different kind of audit, indicators, feedback, time frames, statistical analyses, abundance of involved figures.

This poor comparability of the studies and, in some cases, little information on every specific phase lead us to the impossibility to define a clear conclusion.

Anyway, despite the wide range on interventions and the differences listed above, the results of the A\&F approach in emergency generally show an improvement of the features considered in selected studies, and a statistically significant improvement for the majority of indicators.

The observed improvement refers to: i) clinical outcomes, i.e. the reduction of duration of diabetic ketoacidosis $(p<0.001),{ }^{11}$ the improvement of survival of patients undergoing a cardiac arrest with a shockable initial rhythm after MI $(p=0.02),{ }^{27}$ improvement of neurological recovery of patients survived to a cardiac arrest compared to the historical controls $(81 \%$ vs. $50 \%, p<0.05) ; 27$ ii) appropriateness of clinical test and procedure, i.e. decrease in number of duplicate blood chemistry tests per 100 ED visits $(\mathrm{p}<0.0001)$, reduction in both the daily number of laboratory studies $(-36.3 \% ; \quad \mathrm{p}<0.05),{ }^{18} \quad(\mathrm{p}<0.0001)^{28}$ and in POC tests $(\mathrm{p}<0.0001) ;{ }^{28}$ iii) protocols compliance, i.e. reported by Bentley et $a l .{ }^{8}$ with an $74.3 \%$ improvement of sepsis detection and management, by Hecker et al. ${ }^{13}$ with an increase from 44 to $68 \%$ to adherence to cystitis and pyelonephritis management guidelines, and to confidence by the operators. With regard to this last point, Scott et al. ${ }^{29}$ reported that $100 \%$ of the participants were more likely to identify the indication for Indwelling Urethral Catheterization (IUC) insertion and to use incontinence pads $(72 \%)$, commodes (84\%), straight catheterization (78\%), urinals/bedpans $(89 \%)$, and restrooms $(67 \%)$ as alternatives to IUCs after the intervention; $84 \%$ changed the way they approached bladder management in their practice after the intervention; $83 \%$ were more likely to rethink the use of IUCs among patients with altered mental status, inpatient boarders, and nursing home patients. ${ }^{29}$
Table 3. Different audit and feedback approaches.

\begin{tabular}{lc}
\hline Multiple reports & 15 \\
Educational sessions/meetings & 18 \\
\hline Survey online & 6 \\
Electronic methods & 18 \\
\hline Printed materials & 9 \\
\hline
\end{tabular}

Taylor et al. ${ }^{17}$ reported a high perception of value and satisfaction from participants even if attendance and participation were frequently limited by time barriers and competing clinical duties.

In an A\&F context is essential, as always in clinical settings, the full support of colleagues and a close collaboration to ensure that strategies could be successfully implemented in the complex ED setting, ${ }^{16}$ as it was already highlighted from Ivers et $a l .^{3}$ and Rogers et $a l .{ }^{4}$ Another point of discussion is the fact that A\&F intervention is often carried out after the introduction of something new: that can make more difficult to completely understand the impact of the A\&F intervention compared to the innovation. Probably both of these novelties work together. As described by Pellis et al. ${ }^{27}$ the introduction of Standard Operative Procedures (SOPs) for Target Temperature Management (TTM) and aggressive post-resuscitation care dramatically improved the rate of favorable neurological recovery among patients discharged alive $(81 \%$ vs. $50 \%)$. Moreover, an increase in survival could be achieved by revising and improving the quality of care, by means of periodic audits and continuous professional development courses $(60 \%$ vs. $35 \%))^{27}$ Furthermore, the improvements are often described as persistent like illustrated by Venkatesh et al. for reduction in duplicate blood testing for 11 months since the beginning of the intervention. ${ }^{28}$

The A\&F applied to emergency settings is also influenced by the geographical context. Geographic access to hospital, primary and emergency departments is an important theme for health service policy. ${ }^{35}$ 
The access is influenced by many aspects that include the presence of a National Health Service or private health insurance and public health coverage, various territorial organizations of health service, different geographic realities with specific critical issues, like mountain or rural contexts. In our EASY-NET sub-project we operate in a National Health Service context with many geographic realities, from urban areas to mountain areas that are difficult to access. All of these aspects have to be considered in the evaluation of the emergency settings and so in the A\&F results. Other important emergency settings, often considered for A\&F implementation, are time depending situations, like MI and stroke: we could find them in over a third of our selected articles. Six of them (1/4 of total articles) were conducted in North America.

These studies considered time-depending variables i.e. the time passed from phone call to arrival on the scene (the gold standard is 15 minutes), ${ }^{24}$ interval from call receipt till the call-center operator recognized the necessity of a Telephone cardiopulmonary resuscitation, and the relative times necessary to start instructions by the operator until the by-stander performed the first chest compression. ${ }^{26}$ Almost all of the selected studies were focused on early recognition of the problem and early treatment. For both outcomes, $\mathrm{A} \& \mathrm{~F}$ approach results showed an improvement; i.e.: increase in survival ( $60 \%$ vs. $35 \%),(12 \% \text { vs } 9.1 \%, p=0.02)^{8}$ even after control for potential confounders and risk measures, achievement of successful reanimation by reaching the target temperature within 6 hours $(25.7 \%$ vs $9.0 \%, \mathrm{p}<0.00001),{ }^{25}$ treatment with alteplase within 60 minutes of ED arrival $(1.9 \%$ vs $5.2 \%$; $<<0.01) .{ }^{15}$

The evaluation of time-depending variables is, then, a key aspect that is also fundamental for indicators of essential assistance cares, like Italian "Essential Levels of Care", ${ }^{36}$ which include the services that the Italian National Health Service (SSN) provides to all citizens and concerns collective prevention and public health, district assistance on the territory and hospital assistance, with also A\&F.

The EASY-NET FVG sub-project is focused on MI and stroke and considered different time depending indicators.

We can see the validity of this kind of intervention independently from different A\&F methods. The most used feedback methods are email and frontal lessons or conferences (in about 2/3 of selected studies) and they are often combined together. The EASYNET approach uses all these methods introducing something new: the virtual reality training approach to implement both practice and confidence. We are convinced, as many cited authors, that confidence combined with knowledge are a key stone, because every real change of a behavior came from a deep and personal awareness of the problem and a higher confidence with procedures. ${ }^{37,38}$ This is a concept applied for example in health promotion theories that should be used also in hospitals and it is at the basis of the A\&F approach. A different interesting aspect to take into account is the statistical method used to evaluate the results of the intervention, especially the Interrupted Time Series (ITS), which is the chosen method in EASY-NET FVG sub-project.

ITS analysis is arguably the strongest quasi-experimental research design, particularly useful when a randomized trial is infeasible or unethical ${ }^{39}$ and it is a valuable study design for evaluating the effectiveness of population-level health interventions that have been implemented at a clearly defined point in time; ${ }^{40}$ thus, it's a useful tool to measure quality improvement. The approach usually consists in the construction of a time series of population-level rates for a particular quality improvement focus, and then testing statistically how an outcome rate changes in the time periods before vs time periods after implementation of a designed intervention. ${ }^{39}$ Strengths of ITS include the ability to: i) control secular trends in data, ii) evaluate outcomes using population-level data, clear graphical presentation of results and iii) evaluate both intended and unintended consequences of interventions. ${ }^{39}$

Limitations of ITS include the need for a minimum of 8 time periods before and 8 after an intervention, difficulty in analyzing the independent impact of separate components of a program that are implemented close together in time and existence of a suitable control population. ${ }^{39}$

These limitations can be the reason why ITS was applied only in 3 of our selected studies ${ }^{14,24,28}$ even if we consider ITS a better methodological approach. Other statistical methods used in the majority of our studies are: Fisher exact test, Kruskal Wallis test, Mann Whitney test, ${ }^{27}$ Chi square test, Logistic regression models [8], generalized estimating equation approach. ${ }^{25}$ All of these methods have different limits: for example, a 2-period before-and-after $\mathrm{t}$ test can't control secular trends in data.

The unit of analysis for the comparisons often was the individual patient ${ }^{25}$ while using ITS, individual-level inferences should be avoided when population-level rates are used to evaluate interventions. ${ }^{39}$

Despite the wide range on interventions modality and the poor comparability of the considered studies, the results of the A\&F approach are encouraging and confirm the importance to implement $A \& F$ both in emergency and in other clinical settings.

\section{Conclusions}

The primary aim of this review was to evaluate the diffusion and effectiveness of A\&F in emergency departments and how the process of A\&F can influence medical and, in general, health professionals' behavior in the field of emergency care, either in the hospital or in the out of -hospital interventions.

Starting with the results obtained by Rogers et al., ${ }^{4}$ we performed a review of the literature from January 2014 to December 2019 and we focused on 24 publications. Based on these articles we can affirm that A\&F process in EDs is almost heterogeneous and not standardized either for A\&F approach or for statistical methods.

Despite these limitations, all specific forms of A\&F were considered effective and essential for an increase of quality service in EDs in different emergency situations (sepsis, MI, cardiac arrest, stroke).

\section{References}

1. Gude WT. Understanding and optimizing electronic audit and feedback to improve quality of care. PhD Thesis, University of Amsterdam, 2019. PROVIDE LINK

2. The Quality and Patient Safety Division. A practical guide to clinical audit. National quality improvement Team. August 2013. QPSD-D-029-1. Available from: https:/www.hse.ie/eng/about/who/qid/measurementquality/cli nical-audit/practicalguideclaudit2013.pdf

3. Ivers N, Jamtvedt G, Flottorp S, et al. Audit and feedback: effects on professional practice and healthcare outcomes. Cochrane Database Syst Rev 2012;6:1-227.

4. Rogers RLG, Narvaez Y, Venkatesh AK, et al. Improving emergency physician performance using audit and feedback: a systematic review. Am J Emerg Med 2015;33:1505-14. 
5. Moher D, Liberati A, Tetzlaff J, Altman DG. Preferred reporting items for systematic reviews and meta-analyses: the PRISMA statement. Int J Surg 2010;8:336-41.

6. Singh K, Devarajan R, Mohanan PP, et al. ACS QUIK Investigators. Implementation and acceptability of a heart attack quality improvement intervention in India: a mixed methods analysis of the ACS QUIK trial. Implement Sci 2019;14:12.

7. Aldridge P, Horsley E, Rosettenstein K, et al. What the FLOQ? A quality improvement project to reduce unnecessary paediatric respiratory viral swabs in a peripheral metropolitan hospital. J Paediatr Child Health 2018;54:416-9.

8. Bentley J, Henderson S, Thakore S, et al. Seeking Sepsis in the Emergency Department- Identifying Barriers to Delivery of the Sepsis 6. BMJ Qual Improv Rep 2016;5:u206760.w3983.

9. Bordeleau S, Poitras J, Marceau D, et al. Use of prothrombin complex concentrate in warfarin anticoagulation reversal in the emergency department: a quality improvement study of administration delays. BMC Health Serv Res 2015;15:106.

10. Murphy E, MacGlone S, McGroarty C. A novel approach to improving coagulation sample ordering in an emergency department. BMJ Qual Improv Rep 2015;4:u204785.w2857.

11. Kempegowda P, Coombs B, Nightingale P, et al. Regular and frequent feedback of specific clinical criteria delivers a sustained improvement in the management of diabetic ketoacidosis. Clin Med (Lond) 2017;17:389-94.

12. Khanina A, Cairns KA, McGloughlin S, et al. Improving sepsis care for hospital inpatients using existing medical emergency response systems. Infect Dis Health 2020;25:63-70.

13. Hecker MT, Fox CJ, Son AH, et al. Effect of a stewardship intervention on adherence to uncomplicated cystitis and pyelonephritis guidelines in an emergency department setting. PLoS One 2014;9:e87899.

14. Reznek MA, Barton BA. Improved incident reporting following the implementation of a standardized emergency department peer review process. Int J Qual Health Care 2014;26:27886.

15. Jauch EC, Huang DY, Gardner AJ, Blum JL. Strategies for improving outcomes in the acute management of ischemic stroke in rural emergency departments: a quality improvement initiative in the Stroke Belt. Open Access Emerg Med 2018;10:53-9.

16. Jorgensen SCJ, Yeung SL, Zurayk M, et al. A. Leveraging Antimicrobial Stewardship in the Emergency Department to Improve the Quality of Urinary Tract Infection Management and Outcomes. Open Forum Infect Dis 2018;5:ofy101.

17. Taylor KA, Durrheim DN, Merritt T, et al. Multidisciplinary analysis of invasive meningococcal disease as a framework for continuous quality and safety improvement in regional Australia. BMJ Open Qual 2018;7:e000077.

18. Nazerian P, Vanni S, Fanelli A, et al. Appropriate use of laboratory test requests in the emergency department: a multilevel intervention. Eur J Emerg Med 2019;26:205-11.

19. Scales DC, Golan E, Pinto R, et al. Strategies for Post-Arrest Resuscitation Care Network. Improving Appropriate Neurologic Prognostication after Cardiac Arrest. A Stepped Wedge Cluster Randomized Controlled Trial. Am J Respir Crit Care Med 2016;194:1083-91.

20. Hasan DA, Drennan J, Monger E, et al. Dispatcher assisted cardiopulmonary resuscitation implementation in Kuwait: A before and after study examining the impact on outcomes of out of hospital cardiac arrest victims. Med (Baltimore) 2019;98:e17752.
21. Bloos F, Rüddel H, Thomas-Rüddel D, et al. MEDUSA study group. Effect of a multifaceted educational intervention for anti-infectious measures on sepsis mortality: a cluster randomized trial. Intensive Care Med 2017;43:1602-12.

22. Spencer SP, Karsies T. Audit-and-Feedback and Workflow Changes Improve Emergency Department Care of Critically III Children. Pediatr Qual Saf 2019;4:e128.

23. Stevens MB, Hastings SN, Powers J, et al. Enhancing the Quality of Prescribing Practices for Older Veterans Discharged from the Emergency Department (EQUiPPED): Preliminary Results from Enhancing Quality of Prescribing Practices for Older Veterans Discharged from the Emergency Department, a Novel Multicomponent Interdisciplinary Quality Improvement Initiative. J Am Geriatr Soc 2015;63:1025-9.

24. Oostema JA, Chassee T, Baer W, et al. Brief Educational Intervention Improves Emergency Medical Services Stroke Recognition. Stroke 2019;50:1193-200.

25. Morrison LJ, Brooks SC, Dainty KN, et al. Strategies for PostArrest Care Network. Improving use of targeted temperature management after out-of-hospital cardiac arrest: a stepped wedge cluster randomized controlled trial. Crit Care Med 2015;43:954-64.

26. Bobrow BJ, Spaite DW, Vadeboncoeur TF, et al. Implementation of a Regional Telephone Cardiopulmonary Resuscitation Program and Outcomes After Out-of-Hospital Cardiac Arrest. JAMA Cardiol 2016;1:294-302.

27. Pellis T, Sanfilippo F, Roncarati A, et al. A 4-year implementation strategy of aggressive post-resuscitation care and temperature management after cardiac arrest.Resuscitation. 2014 Sep;85(9):1251-6.

28. Venkatesh AK, Hajdasz D, Rothenberg C, et al. Reducing Unnecessary Blood Chemistry Testing in the Emergency Department: Implementation of Choosing Wisely. Am J Med Qual 2018;33:81-5.

29. Scott RA, Oman KS, Makic MB, et al. Reducing indwelling urinary catheter use in the emergency department: a successful quality-improvement initiative. J Emerg Nurs 2014;40:237-44.

30. van de Ridder JM, Stokking KM, McGaghie WC, ten Cate OT. What is feedback in clinical education?. Med Educ 2008;42:189-97.

31. Thomas JD, Arnold RM. Giving feedback. J Palliat Med 2011;14:233-9.

32. Gude WT, Brown B, van der Veer SN, et al. Clinical performance comparators in audit and feedback: a review of theory and evidence. Implement Sci 2019;14:39.

33. Ullman AJ, Ray-Barruel G, Rickard CM, Cooke M. Clinical audits to improve critical care: Part 1 Prepare and collect data. Aust Crit Care 2018;31:101-5.

34. Ray-Barruel G, Ullman AJ, Rickard CM, Cooke M. Clinical audits to improve critical care: Part 2: Analyse, benchmark and feedback. Aust Crit Care 2018;31:106-9.

35. Fone DL, Christie S, Lester N. Comparison of perceived and modelled geographical access to accident and emergency departments: a cross-sectional analysis from the Caerphilly Health and Social Needs Study Int J Health Geogr 2006;5:16.

36. Decree by the President of the Italian Council of Ministers. [Definizione e aggiornamento dei livelli essenziali di assistenza, di cui all'articolo 1, comma 7, del decreto legislativo 30 dicembre 1992, n. 502. (17A02015). (G.U. Serie Generale, n. 65 del 18 marzo 2017).] Available from: http://www.trovanorme.salute.gov.it/norme/dettaglioAtto?id= 58669 \& completo=true [Website in Italian].

37. Boling B, Hardin-Pierce M. The effect of high-fidelity simula- 
tion on knowledge and confidence in critical care training: An integrative review. Nurse Educ Pract 2016;16:287-93.

38. Pulford BD, Colman AM, Buabang EK, Krockow EM. The persuasive power of knowledge: Testing the confidence heuristic. J Exp Psychol Gen 2018;147:1431-44.

39. Penfold RB, Zhang F. Use of Interrupted Time Series Analysis in Evaluating Health Care Quality Improvements. Acad Pediatr 2013;13:S38-44.

40. Bernal JL, Cummins S, Gasparrini A. Interrupted time series regression for the evaluation of public health interventions: a tutorial. Int J Epidemiol 2017;46:348-55. 